\title{
En falla cardíaca, la recuperación de la frecuencia cardíaca después de la caminata de 6 minutos es mejor indicador pronóstico que la distancia recorrida
}

\author{
In heart failure, recovery of the heart rate after the six-minute walk is a \\ better prognostic indicator than the distance covered
}

Gustavo Adolfo Hincapié Díaz ${ }^{(1)}$

Rev Colomb Neumol 2013; 25 (1):60-62

\section{REFERENCIA}

Lawrence PC, Ross Arena VL, Bandera F, Lavie CF, Guazzi M. Heart rate recovery after the $6 \mathrm{~min}$ walk test rather than distance ambulated is a powerful prognostic indicator in heart failure with reduced and preserved ejection fraction: a comparison with cardiopulmonary exercise testing. Eur J Heart Failure. 2013; 15: 519-527.

\section{PREGUNTA}

¿Cuál es la utilidad pronóstica de la recuperación de la frecuencia cardiaca después de una evaluación funcional submáxima como la caminata de 6 minutos (C6M) en pacientes con falla cardiaca?

\section{DISEÑO}

Estudio de cohorte prospectiva. Análisis de diferencias en supervivencia.

\section{PERIODO DE SEGUIMIENTO}

Desde junio de 1999 hasta diciembre de 2008.

\section{LUGAR}

Centro de referencia de falla cardíaca, Hospital San Paolo, Milán, Italia.

\section{PACIENTES}

258 pacientes con diagnóstico de falla cardíaca (edad promedio 62 años, 75\% hombres) con clase funcional de la NYHA II - III. Se incluyeron pacientes con fracción de eyección reducida y fracción de eyección preservada. A todos se les realizó C6M y prueba de ejercicio cardiopulmonar integrada.

\section{INTERVENCIÓN}

- Caminata de 6 minutos: el resultado fue dicotomizado en más de 300 metros y menos de 300 metros. Se les midió la frecuencia cardiaca inmediatamente después del ejercicio y al minuto. La recuperación de la frecuencia cardiaca se definió como la diferencia entre la frecuencia cardiaca al final del ejercicio y al minuto luego de reposo.

- Ejercicio cardiopulmonar: hasta llegar a consumo de oxígeno $\left(\mathrm{VO}_{2}\right)$ máximo. Se midió la frecuencia

\footnotetext{
(1) Médico Internista - Neumólogo. Profesor asociado Universidad Militar Nueva Granada. Coordinador Servicio de Neumología, Hospital Militar Central, Bogotá, Colombia.
}

Correspondencia: Gustavo Adolfo Hincapié Díaz. Correo electrónico: gahincapie@gmail.com

Recibido: 20-05-2013. Aceptado: 25-05-2013. 
cardiaca y la recuperación de ésta como se definió previamente.

- Ecocardiograma: se calculó la fracción de eyección con el método de Simpson.

Finalizaba el seguimiento al ocurrir un evento catastrófico de origen cardiaco, como muerte o necesidad de trasplante de urgencia.

\section{RESULTADOS}

Durante el periodo de seguimiento se detectaron 50 eventos cardiacos mayores. Los resultados con el análisis de regresión univariado, mostraron que la recuperación de la frecuencia cardiaca después de la $\mathrm{C} 6 \mathrm{M}$ y la prueba de ejercicio cardiopulmonar integrada, fue un predictor de desenlace fatal. El análisis de regresión multivariado indicó que la recuperación de la frecuencia cardiaca después de la C6M y la prueba de ejercicio cardiopulmonar integrada fue el predictor de sobrevida de mayor poder. No hubo diferencias en pacientes con fracción de eyección reducida en comparación con fracción de eyección preservada.

La sensibilidad y especificidad de la recuperación de la frecuencia cardiaca después de la C6M, fue de 0,94 y 0,55 respectivamente. La sensibilidad y especificidad de la recuperación de la frecuencia cardiaca luego de la prueba de ejercicio cardiopulmonar integrada fue de 0,88 y 0,74 respectivamente. En la figura 1 se muestran las curvas ROC que comparan la recuperación de la frecuencia cardiaca con distancia recorrida y la recuperación de la frecuencia cardiaca en los dos métodos de ejercicio.

En la figura 2 se observan las diferencias en sobrevida en la curva de Kaplan Meier, entre quienes tenían recuperación de la frecuencia cardiaca mayor a 12 en comparación con menor a 12 tanto en la C6M como en la prueba de ejercicio cardiopulmonar integrada.

\section{CONCLUSIÓN}

La recuperación de la frecuencia cardiaca después de la $\mathrm{C} 6 \mathrm{M}$ en un indicador de pronóstico similar al de la recuperación de la frecuencia cardiaca después de ejercicio máximo. En estudios posteriores se sugiere reemplazar la recuperación de la frecuencia cardiaca después de la C6M por la distancia recorrida en la misma.

\section{COMENTARIO}

La distancia recorrida en la C6M es la medición más importante de esta prueba diagnóstica. De hecho, caminar menor distancia que la esperada para cada paciente, se relaciona con mayor intolerancia

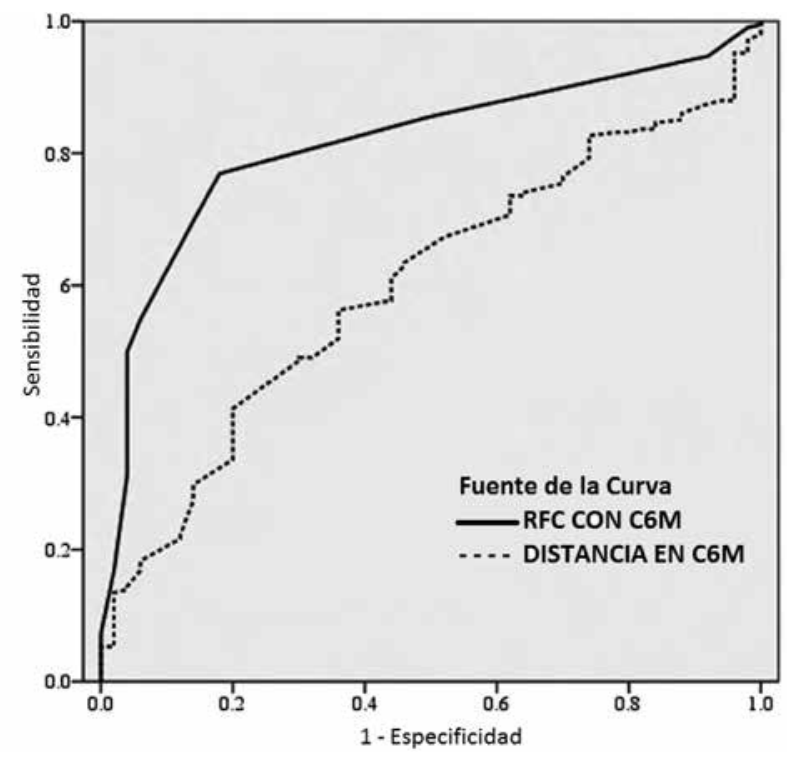

A.

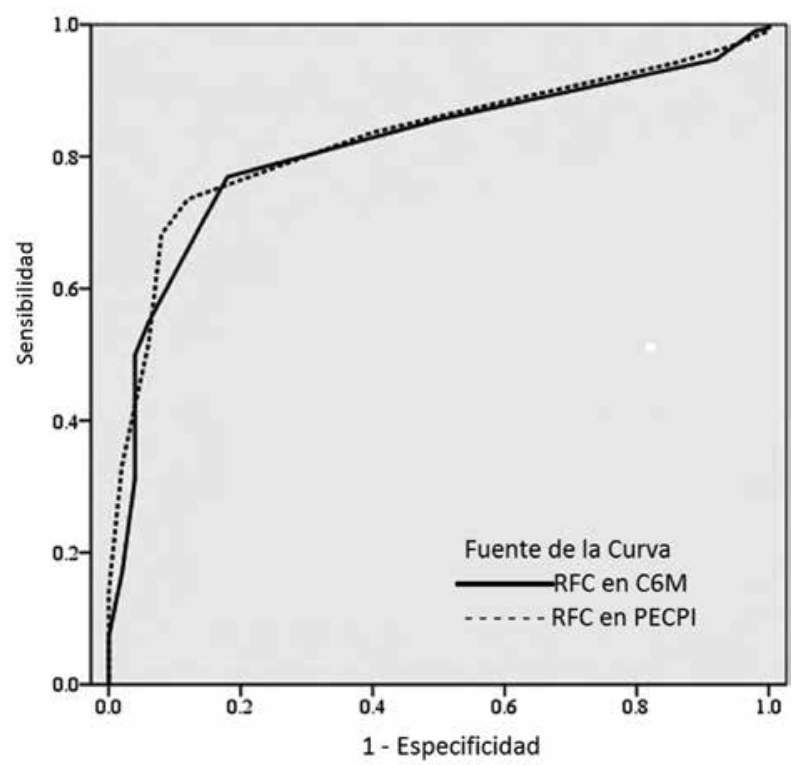

B.

Figura 1. Curva ROC A: recuperación de la frecuencia cardiaca en la C6M vs. distancia recorrida. B: recuperación de la frecuencia cardiaca en la C6M y en la prueba de ejercicio cardiopulmonar integrada. Adaptada de referencia 5. 


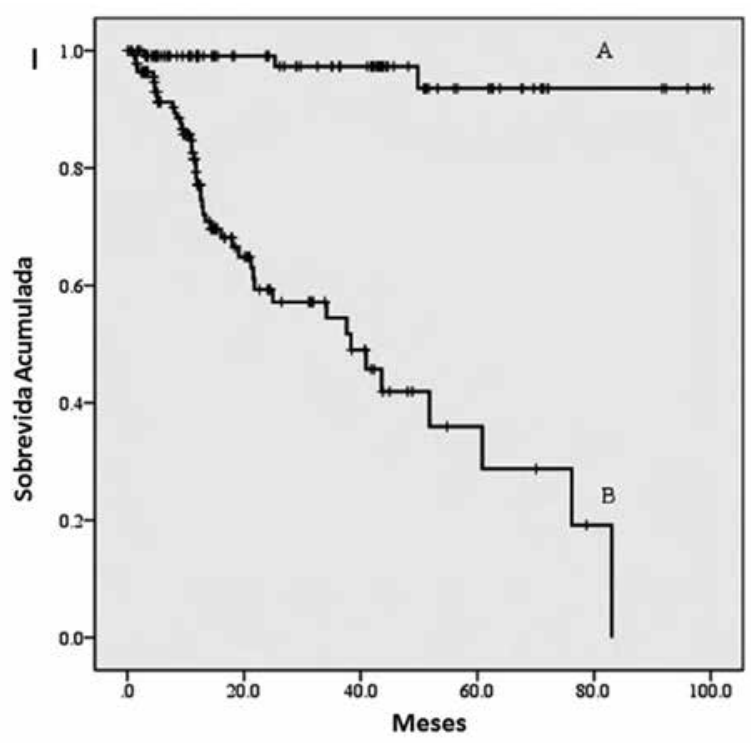

A.

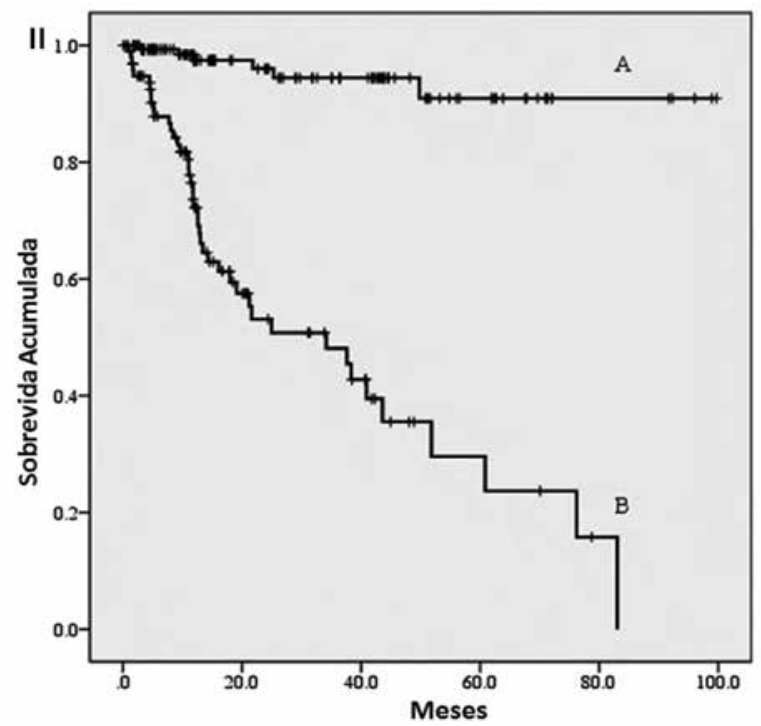

B.

Figura 2. Análisis de Kaplan Meier, para l; recuperación de la frecuencia cardiaca en C6M y II recuperación de la frecuencia cardiaca en la prueba de ejercicio cardiopulmonar integrada. A: recuperación de la frecuencia cardiaca mayor a 12. B: recuperación de la frecuencia cardiaca menor a 12. Adaptada de referencia 5.

al ejercicio y por lo tanto con mayor riesgo de enfermedad. Incluso, en enfermedad pulmonar obstructiva crónica es un determinante de mortalidad el tener una distancia recorrida inferior a lo normal, y cuanto más baja es la distancia mayor la mortalidad.

Lo mismo ocurre en falla cardiaca; sin embargo, al comparar la distancia recorrida en metros, con la recuperación de la frecuencia cardiaca luego del reposo, es esta última medición la que tiene mejor poder como indicador de pronóstico, a tal punto que los autores sugieren cambiar la variable distancia por la recuperación de la frecuencia cardiaca, al menos en falla cardiaca (5).

\section{CONFLICTOS DE INTERESES}

El autor no declara algún conflicto de interés.

\section{BIBLIOGRAFÍA}

1. Huang $\mathrm{PH}$, Leu HB, Chen JW, Lin SJ. Heart rate recovery after exercise and endotelial function. Two important factors to predict cardiovascular events. Prev Cardiol. 2005; 8: 167-170.

2. Cole CR, Blackstone EH, Pashkow FJ, Snader CE, Lauer MS. Heart-rate recovery immediately after exercise as a predictor of mortality. N Engl J Med. 1999; 341: 1351-1357.

3. Nishime EO, Cole CR, Blackstone EH, Pashkow FJ, Lauer MS. Heart rate recovery and treadmill exercise score as predictors of mortality in patients referred for exercise ECG. JAMA. 2000; 284: 1392-1398.

4. Brubaker PH, Kitzman DW. Chronotropic incompetence: causes, consequences, and management. Circulation. 2011; 123: 1010-1020.

5. Lawrence PC, Ross Arena VL, Bandera F, Lavie CF, Guazzi M. Heart rate recovery after the 6 min walk test rather than distance ambulated is a powerful prognostic indicator in heart failure with reduced and preserved ejection fraction: a comparison with cardiopulmonary exercise testing. Eur J Heart Failure. 2013; 15: 519-527. 